\title{
Hölder properties of local times for fractional Brownian motions
}

\author{
D. Baraka - T. Mountford • Y. Xiao
}

Published online: 24 December 2008

(C) Springer-Verlag 2008

\begin{abstract}
We study the local times of fractional Brownian motions for all temporal dimensions, $N$, spatial dimensions $d$ and Hurst parameters $H$ for which local times exist. We establish a Hölder continuity result that is a refinement of Xiao (Probab Th Rel Fields 109:129-157, 1997). Our approach is an adaptation of the more general attack of Xiao (Probab Th Rel Fields 109:129-157, 1997) using ideas of Baraka and Mountford (1997, to appear), the principal result of this latter paper is contained in this article.
\end{abstract}

Keywords Local times · Fractional Brownian motion $\cdot$ Law of the iterated logarithm

\section{Introduction and main results}

In this paper we wish to apply and generalise techniques of Baraka and Mountford (1997) (see also Mountford 2007; Mountford and Nualart 2004; Xiao 2007) to refine results of Xiao (1997) for the local times of fractional Brownian motions.

Research of Y. Xiao is partially supported by NSF grant DMS-0706728.

D. Baraka $(\varangle) \cdot$ T. Mountford

Institut de Mathématiques, École Polytechnique Fédéralede Lausanne,

Station 8, 1015 Lausanne, Switzerland

e-mail: driss.baraka@epfl.ch

T. Mountford

e-mail: thomas.mountford@epfl.ch

Y. Xiao

Department of Statistics and Probability, Michigan State University,

East Lansing, MI 48824, USA

e-mail:xiao@stt.msu.edu 
To recall, fractional Brownian motions of parameter $0<H<1$ are centred, path continuous, real-valued Gaussian processes

$$
Y=\left\{Y(\underline{t}), \underline{t} \in \mathbb{R}^{N}\right\} \rightarrow \mathbb{R}
$$

with $Y(0)=0$ and covariance function

$$
\operatorname{Cov}(Y(\underline{t}), Y(\underline{s}))=\frac{1}{2}\left(|\underline{t}|^{2 H}+|\underline{s}|^{2 H}-|\underline{t}-\underline{s}|^{2 H}\right)
$$

where $|\cdot|$ is the Euclidean norm in $\mathbb{R}^{N}$. Note that for $N=1, H=\frac{1}{2}$, we have classical Brownian motion. This fully specifies the distribution of the process (see, e.g., Adler 1981; Samorodnitsky and Taqqu 1994).

We can then form the $d$-dimensional fractional Brownian motion $\underline{X}=\{\underline{X}(\underline{t}), \underline{t} \in$ $\mathbb{R}^{N}$ \} by adjoining $d$ independent copies of $Y$

$$
\underline{X}(\underline{t})=\left(X_{1}(\underline{t}) \cdots, X_{d}(\underline{t})\right), \quad \forall \underline{t} \in \mathbb{R}^{N} .
$$

It is sometimes called an $(N, d)$ fractional Brownian motion with index $H$.

The standard methods of Geman and Horowitz (1980) can be used to show the existence of local times in the case $N>d H$; where for $I \subseteq \mathbb{R}^{N}$ bounded (here and in the following, an interval in $\mathbb{R}^{N}$ will simply be the Cartesian product of one dimensional intervals) and $\underline{x} \in \mathbb{R}^{d}$

$$
L_{\underline{x}}(I)=\lim _{\epsilon \downarrow 0} \frac{1}{C_{d} \epsilon^{d}} \lambda_{N}\{\underline{t} \in I:|\underline{X}(\underline{t})-\underline{x}|<\epsilon\},
$$

where $\lambda_{N}$ is Lebesgue measure on $N$-dimensional Euclidean space and $C_{d}$ is the volume of the unit ball in $d$-dimensional Euclidean space. On the other hand, if $d H \geq N$, the results in Talagrand $(1995,1998)$ imply that for every $I \subseteq \mathbb{R}^{N}, \lambda_{d}(\underline{X}(I))=0$ almost surely. Hence $\underline{X}$ has no local times on $I$. Hence, an $(N, d)$ fractional Brownian motion $\underline{X}$ has local times if and only if $N>d H$. From this point on we assume that this inequality holds.

Of course much is known, and has been known of the properties of these local times for Brownian motion. For fractional Brownian motions, with fewer tools available, things are less complete. Nevertheless one has (see Kasahara et al. 1999; Xiao 1997, 2006)

$$
\begin{gathered}
\limsup _{x \uparrow \infty} \frac{-\log \mathbb{P}\left(L_{\underline{0}}\left([0,1]^{N}\right)>x\right)}{x^{\frac{N}{d H}}}<C_{1.1}<\infty, \\
\liminf _{x \uparrow \infty} \frac{-\log \mathbb{P}\left(L_{\underline{0}}\left([0,1]^{N}\right)>x\right)}{x^{\frac{N}{d H}}}>C_{1.2}>0,
\end{gathered}
$$


at least for $N=1$ in the case of (1.5). For higher dimensions (i.e., $N>1$ ), see Sect. 2 , though this is implicit in Xiao (2006). Recently, Mountford et al. (2008), based on this result, showed that in fact there exists a constant $C_{1.3}=C(N, d, H)$ so that

$$
\lim _{x \uparrow \infty} \frac{-\log \mathbb{P}\left(L_{\underline{0}}\left([0,1]^{N}\right)>x\right)}{x^{\frac{N}{d H}}}=C_{1.3} .
$$

This enabled Xiao (1997) to establish (indeed a wider class of Gaussian processes was considered),

(A) For $\phi$ the function $t^{N-d H}\left(\log \log \left(\frac{1}{t}\right)\right)^{\frac{H d}{N}}$ and $I$ an interval of $\mathbb{R}^{N}$

$$
L_{\underline{0}}(I)>0 \text { implies } m_{\phi}\{\underline{t}: \underline{t} \in I, \quad \underline{X}(\underline{t})=\underline{0}\}>0,
$$

(B) $m_{\phi}\{\underline{t} \in I: \underline{X}(\underline{t})=\underline{0}\} \leq C L_{0}(I)$ for some finite constant $C$. Here $m_{\phi}$ denotes the Hausdorff measure with respect to $\phi$; see Rogers (1998).

(C) If for interval $B \subseteq \mathbb{R}^{N}$ one defines $L^{*}(B)=\sup _{\underline{x}} L_{\underline{x}}(B)$, then for all $T>0$ there exists $C<\infty$ so that

$$
\sup _{\substack{|B| \downarrow 0 \\ B \subseteq[-T, T]^{N}}} \frac{L^{*}(B)}{\psi(|B|)} \leq C
$$

where $\psi(x)=x^{N-d H}\left(\log \frac{1}{x}\right)^{\frac{H d}{N}}$ and $|B|$ denotes the diameter of $B$.

A recent paper (Baraka and Mountford 2008) showed, in fact, that the results of (A) and (B) could be improved to

Theorem 1 There exists a constant $C_{1.4}=C(N, d, H) \in(0, \infty)$ so that for $N>$ $d H$, for all finite interval I

$$
m_{\phi}\{\underline{t}: \underline{t} \in I, \underline{X}(\underline{t})=\underline{0}\}=C_{1.4} L_{\underline{0}}(I) .
$$

And Baraka and Mountford (1997) considered fractional Brownian motions with $N=1$ and showed.

Theorem 2 For $\underline{X}=\{\underline{X}(t), t \in \mathbb{R}\}$ a d-dimensional fractional Brownian motion with index $H$

$$
\limsup _{t \downarrow 0} \frac{L_{\underline{0}}([0, t])}{t^{1-d H}\left(\log \log \left(\frac{1}{t}\right)\right)^{d H}}=C_{1.5} \in(0, \infty) .
$$

We will develop these ideas to show this result in all temporal dimensions. 
Theorem 3 For $\underline{X}=\left\{\underline{X}(\underline{t}), \underline{t} \in \mathbb{R}^{N}\right\}$ a $(N, d)$ fractional Brownian motion with index $H$, there exists a finite constant $C_{1.6}=C(N, d, H)$ such that

$$
\limsup _{t \downarrow 0} \frac{L_{\underline{0}}\left([0, t]^{N}\right)}{t^{N-d H}\left(\log \log \left(\frac{1}{t}\right)\right)^{\frac{d H}{N}}}=C_{1.6}
$$

And also show

Theorem 4 For $\underline{X}=\left\{\underline{X}(\underline{t}), \underline{t} \in \mathbb{R}^{N}\right\} a(N, d)$ fractional Brownian motion with index $H$ and $T>0$ fixed, there exists a finite constant $C_{1.7}=C(N, d, H)$ such that

$$
\lim _{\delta \downarrow 0} \sup _{\substack{B \subset[-T, T]^{N} \\|B|=\delta}} \frac{L^{*}(B)}{\delta^{N-d H}\left(\log \frac{1}{\delta}\right)^{\frac{d H}{N}}}=C_{1.7} .
$$

In both instances the basic question is how to create enough independent or essentially independent trials. This is done via the exploitation of a white noise representation of the fractional Brownian motion. We "create" independence via substituting independent white noise over secondary regions: we use the representation (see, e.g., Embrechts and Maejima 2002 or Samorodnitsky and Taqqu 1994)

$$
X_{i}(\underline{t})=C_{N, H} \int_{\mathbb{R}^{N}}\left(\frac{1}{|\underline{x}-\underline{t}|^{\frac{N}{2}-H}}-\frac{1}{|\underline{x}|^{\frac{N}{2}-H}}\right) d \dot{W}_{i}(\underline{x})
$$

for $\dot{W}_{i}$ independent white noise on $\mathbb{R}^{N}$. A useful comparison process is

$$
X_{i}^{\prime}(\underline{t})=C_{N, H} \int_{\mathbb{R}^{N}}\left(\frac{1}{|\underline{x}-\underline{t}|^{\frac{N}{2}-H}}-\frac{1}{|\underline{x}|^{\frac{N}{2}-H}}\right) d \dot{W}_{i}^{\prime}(\underline{x})
$$

for

$$
\begin{aligned}
\dot{W}_{i}^{\prime}(\underline{x}) & =\dot{W}_{i}(\underline{x}) \quad \text { for } \underline{x} \in I \\
& =\dot{Z}_{i}(\underline{x}) \quad \text { elsewhere, }
\end{aligned}
$$

where $\dot{Z}_{i}$ is a white noise independent of $\underline{\dot{W}}$ and of $\dot{Z}_{j}$ for $j \neq i$. Here typically we take $I$ to be an interval but in Sect. 3 we take $I$ to be an interval with an inner subinterval removed.

If we are interested in $\underline{X}(\underline{t}), \underline{t} \in\left[\underline{t}_{0}, \underline{t}_{0}+h\right]$ (here we adopt the convention for $\underline{t}_{0}=\left(\left(t_{0}\right)_{1},\left(t_{0}\right)_{2}, \ldots,\left(t_{0}\right)_{N}\right), h \in \mathbb{R}_{+}$that $\underline{t}_{0}+h$ is the element of $\mathbb{R}^{N}$ such that $\underline{t}_{0}+h=\left(\left(t_{0}\right)_{1}+h,\left(t_{0}\right)_{2}+h, \ldots,\left(t_{0}\right)_{N}+h\right)$ and $\left[\underline{t}_{0}, \underline{t}_{0}+h\right]=\left\{\underline{t}: \forall i,\left(t_{0}\right)_{i} \leq t_{i} \leq\right.$ $\left.\left.\left(t_{0}\right)_{i}+h\right\}\right)$ and we take comparison process $\underline{X}^{\prime}(\underline{t})$ with interval $I=\left[\underline{t}_{0}-V, \underline{t}_{0}+h+V\right]$, where $V>>h$, then for every $1 \leq i \leq d$ and all $\underline{s}, \underline{t} \in\left[\underline{t}_{0}, \underline{t}_{0}+h\right]$ 


$$
\begin{aligned}
& \operatorname{Cov}\left(X_{i}(\underline{t})-X_{i}\left(\underline{t}_{0}\right),\left(X_{i}(\underline{s})-X_{i}\left(\underline{t}_{0}\right)\right)-\left(X_{i}^{\prime}(\underline{s})-X_{i}^{\prime}\left(\underline{t}_{0}\right)\right)\right) \\
& =\mathbb{E}\left[\left(X_{i}(\underline{t})-X_{i}\left(\underline{t}_{0}\right)\right)\left(\left(X_{i}(\underline{s})-X_{i}\left(\underline{t}_{0}\right)\right)-\left(X_{i}^{\prime}(\underline{s})-X_{i}^{\prime}\left(\underline{t}_{0}\right)\right)\right)\right] \\
& =C_{N, H}^{2} \int_{I^{c}}\left(\frac{1}{\mid \underline{x}-\underline{t}^{\frac{N}{2}-H}}-\frac{1}{\left|\underline{x}-\underline{t}_{0}\right|^{\frac{N}{2}-H}}\right)\left(\frac{1}{\mid \underline{x}-\underline{s}^{\frac{N}{2}-H}}-\frac{1}{\left|\underline{x}-\underline{t}_{0}\right|^{\frac{N}{2}-H}}\right) d \underline{x} \\
& \leq C \frac{\left|\underline{t}-\underline{t}_{0}\right|\left|\underline{s}-\underline{t}_{0}\right|}{V^{2-2} H}
\end{aligned}
$$

for universal $C$. Using this inequality one can show that

$$
\begin{aligned}
& \mathbb{E} {\left[\left(L_{\underline{\underline{0}}}^{\underline{X}}([\underline{0}, \underline{0}+h])-L_{\underline{\underline{0}}}^{\underline{X^{\prime}}}([\underline{0}, \underline{0}+h])\right)^{2}\right] } \\
&=2 \int_{[0,1]^{N}} \int_{[0,1]^{N}} d \underline{s} d \underline{t} \quad\left(P_{\underline{s}, \underline{t}}(\underline{0}, \underline{0})-P_{\underline{s}, \underline{t}}^{\prime}(\underline{0}, \underline{0})\right)
\end{aligned}
$$

is small, for $P_{\underline{s}, \underline{t}}(\underline{0}, \underline{0})$ the joint density at $(\underline{0}, \underline{0})$ for $(\underline{X}(\underline{t}), \underline{X}(\underline{s}))$ and $P_{s, t}^{\prime}(\underline{0}, \underline{0})$ that at $(\underline{0}, \underline{0})$ for $\left(\underline{X}(\underline{t}), \underline{X}^{\prime}(\underline{s})\right)$ (and so for $\left(\underline{X}(\underline{s}), \underline{X}^{\prime}(\underline{t})\right)$.

This we will see, is sufficient to show Theorem 3. However, for Theorem 4 we will require superior bound for this difference.

In Sect. 2 we prove (1.5) for $N>1$. This result is used to prove Theorem 3 in Sect. 3. In Sect. 4 we show a LND property for a certain process. This will be used, in Sect. 5, to found a bound on local times differences between the comparison and original process. Section 6, using some results of Sects. 4 and 5, is devoted to the proof of the analogous version of (1.6) for the supremum of the local time. Finally, in Sect. 7, we prove Theorem 4 using the result of Sect. 5.

Unspecified positive and finite constants will be denote by $C, C_{1}, C_{2}, \ldots$ which may have different values from line to line. Specific constants in section $i$ will be denoted by $C_{i .1}, C_{i .2}, \ldots$.

\section{Tail probability of local times}

In this section, we show (1.4) and (1.5) and generalize the result of Kasahara et al. (1999) to $(N, d)$ fractional Brownian motion with $N>1$. We prove that

Theorem 5 If $\underline{X}=\left\{\underline{X}(\underline{t}), \underline{t} \in \mathbb{R}^{N}\right\}$ is a $(N, d)$ fractional Brownian motion with index $0<H<1$, then

$$
-\log \mathbb{P}\left(L_{\underline{0}}\left([0,1]^{N}\right)>x\right) \approx x^{N / d H} \text { as } x \rightarrow \infty .
$$

Where $f \approx g$ means $0<\liminf _{x \rightarrow \infty} f(x) / g(x) \leq \limsup _{x \rightarrow \infty} f(x) / g(x)<\infty$. This result is necessary to prove that the limit in (1.6) is strictly between 0 and $\infty$, see Mountford et al. (2008). 
Our basic tool for the proof is the following Tauberian theorem obtained by Kasahara (1978, Theorem 4).

Theorem 6 Let $Z$ be a positive random variable and let $\phi(\cdot)$ be a function which varies regularly at $\infty$ with exponent $0<\alpha<1$. Then

$$
-\log \mathbb{P}(Z>\phi(x)) \approx x \text { as } x \rightarrow \infty
$$

holds if and only if

$$
\mathbb{E}\left[Z^{n}\right]^{1 / n} \approx \phi(n) \text { as } n \rightarrow \infty
$$

Let $\phi(n)=n^{d H / N}$. Since $\phi^{-1}(x)=x^{N / d H}$, then Theorem 5 is equivalent to

\section{Theorem 7}

$$
\mathbb{E}\left[L_{\underline{0}}\left([0,1]^{N}\right)^{n}\right]^{1 / n} \approx n^{d H / N} \text { as } n \rightarrow \infty
$$

For the upper bound we have the result of Xiao (1997) where it was proved that there exists a positive finite constant $C$ such that

$$
\mathbb{E}\left[L_{\underline{0}}\left([0,1]^{N}\right)^{n}\right] \leq C^{n}(n !)^{d H / N}
$$

Using the Stirling formula,

$$
n ! \approx \sqrt{2 \pi n} \frac{n^{n}}{e^{n}} \text { as } n \rightarrow \infty
$$

we obtain the following

Proposition 1 Let $\underline{X}=\left\{\underline{X}(\underline{t}), \underline{t} \in \mathbb{R}^{N}\right\}$ be a d-dimensional fractional Brownian motion with index $0<H<1$, then there exists a positive finite constant $C$ such that

$$
\mathbb{E}\left[L_{\underline{0}}\left([0,1]^{N}\right)^{n}\right]^{1 / n} \leq C n^{d H / N} \text { as } n \rightarrow \infty
$$

For the lower bound, we start with the following lemma, whose proof will be given at the end of this section,

Lemma 1 Let $V \sim \operatorname{Binom}\left(n, n^{\frac{1-N}{N}}\right)$, so

$$
\mathbb{P}\left(V-n^{1 / N} \leq-n^{1 /(2 N)} x\right) \leq e^{-x^{2} / 4}
$$

Since $X_{1}, \ldots, X_{d}$ are independent copies of $Y$, we can write, see Geman and Horowitz (1980), 


$$
\begin{aligned}
& \mathbb{E}\left[L_{\underline{0}}\left([0,1]^{N}\right)^{n}\right]=\int_{[0,1]^{N}} d \underline{t}_{1} \ldots \int_{[0,1]^{N}} d \underline{t}_{n} p_{\underline{t}_{1}, \ldots, \underline{t}_{n}}(\underline{0}, \ldots, \underline{0}) \\
& =(2 \pi)^{n d / 2} \int_{[0,1]^{N}} d \underline{t}_{1} \ldots \int_{[0,1]^{N}} d \underline{t}_{n} \operatorname{det} \operatorname{Cov}\left[Y\left(\underline{t}_{1}\right), \ldots, Y\left(\underline{t}_{n}\right)\right]^{-d / 2},
\end{aligned}
$$

where $p_{\underline{t}_{1}, \ldots, \underline{t}_{n}}(\underline{0}, \ldots, \underline{0})$ denote the density at $(\underline{0}, \ldots, \underline{0})$ of the random vector $\left(\underline{X}\left(\underline{t}_{1}\right), \ldots, \underline{X}\left(\underline{t}_{n}\right)\right)$ and $\operatorname{det} \operatorname{Cov}\left[Y\left(\underline{t}_{1}\right), \ldots, Y\left(\underline{t}_{n}\right)\right]$ the determinant of the covariance matrix of the random vector $\left(Y\left(\underline{t}_{1}\right), \ldots, Y\left(\underline{t}_{n}\right)\right)$. For any permutation $\pi$ for $\{1,2, \ldots, n\}$

$$
\begin{aligned}
\operatorname{det} \operatorname{Cov}\left[Y\left(\underline{t}_{1}\right), \ldots, Y\left(\underline{t}_{n}\right)\right] & =\operatorname{Var}\left(Y\left(\underline{t}_{\pi(1)}\right)\right) \prod_{i=2}^{n} \operatorname{Var}\left(Y\left(\underline{t}_{\pi(i)}\right) \mid Y\left(\underline{t}_{\pi(1)}\right), \ldots, Y\left(\underline{t}_{\pi(i-1)}\right)\right) \\
& \leq \operatorname{Var}\left(Y\left(\underline{t}_{\pi(1)}\right)\right) \prod_{i=2}^{n} \operatorname{Var}\left(Y\left(\underline{t}_{\pi(i)}\right) \mid Y\left(\underline{t}_{\pi(i-1)}\right)\right) .
\end{aligned}
$$

To simplify, we denote $\pi(i)=(i)$ when $\pi$ is given.

We will use a probabilistic interpretation of the integral in (2.6) as

$$
\mathbb{E}\left[\operatorname{det} \operatorname{Cov}\left(Y\left(\underline{T}_{1}\right), \ldots, Y\left(\underline{T}_{n}\right)\right)^{-d / 2}\right]
$$

where the $n$ points $\underline{T}_{i}=\left(T_{i}^{1}, \ldots, T_{i}^{N}\right), i=1, \ldots, n$ are uniformly distributed on $[0,1]^{N}$. So we divide $[0,1]^{N}$ in $n$ subcubes with side of length $n^{-1 / N}$. The subcubes are

$$
\begin{aligned}
& {\left[(\underline{i}-1) n^{-1 / N}, \underline{i} n^{-1 / N}\right]} \\
& \quad=\left[\left(i_{1}-1\right) n^{-1 / N} ; i_{1} n^{-1 / N}\right] \times \cdots \times\left[\left(i_{N}-1\right) n^{-1 / N} ; i_{N} n^{-1 / N}\right]
\end{aligned}
$$

with $i_{j} \in\left\{1,2, \ldots, n^{1 / N}\right\}, 1 \leq j \leq N$. We expect about one point in each subcube. By fixing all $i_{j}, 2 \leq j \leq N$ and moving $i_{1}$ along $\left[1,2, \ldots, n^{1 / N}\right]$, we get $n^{\frac{N-1}{N}}$ columns each containing $n^{1 / N}$ subcubes. The first column $C_{1}$ is

$$
[0 ; 1] \times\left[0 ; n^{-1 / N}\right] \times \cdots \times\left[0 ; n^{-1 / N}\right]
$$

Let $\underline{V}=\left(V_{1}, \ldots, V_{n} \frac{N-1}{N}\right)$, where $V_{k}$ is the number of points in the column $C_{k}, k=$ $1, \ldots, n^{\frac{N-1}{N}} . V_{k}$ has a binomial distribution of parameters $\left(n, n^{\frac{1-N}{N}}\right)$ and if

$$
A_{k}=\left\{V_{k}-n^{1 / N} \geq-n^{1 /(2 N)} \log n\right\} \text { and } A=\cap_{k} A_{k}
$$


we have by Lemma 1 that $\mathbb{P}\left(A_{k}^{c}\right) \leq e^{-\frac{1}{4} \log ^{2} n}$. Hence

$$
\begin{aligned}
\mathbb{P}(A) & =1-\mathbb{P}\left(\cup_{k=1}^{n^{1-1 / N}} A_{k}^{c}\right) \\
& \geq 1-n^{1-1 / N} e^{-\frac{1}{4} \log ^{2} n} \\
& =1-n^{\frac{N-1}{N}-\frac{1}{4} \log n} \geq \frac{1}{2} \text { for } n \text { big enough. }
\end{aligned}
$$

Since for all $i, \operatorname{Var}\left(Y\left(\underline{T}_{(i)}\right) \mid Y\left(\underline{T}_{(i-1)}\right)\right) \leq 1$ and in each column the same argument holds, then we can consider only the column $C_{1}$. It will be enough from (2.6)-(2.8) and (2.10) to understand

$$
\mathbb{E}\left[\prod_{i=1}^{V_{1}} \frac{1}{\operatorname{Var}\left(Y\left(\underline{T}_{(i)}\right) \mid Y\left(\underline{T}_{(i-1)}\right)\right)^{d / 2}} \mid \underline{V}\right]^{n^{1-1 / N}} \quad \text { on event } A .
$$

We choose the permutation $\pi$ such that

$$
\underline{T}_{(i)} \in C_{1} \Leftrightarrow i \leq V_{1} \text { and } 0=T_{(0)}^{1} \leq T_{(1)}^{1} \leq T_{(2)}^{1} \leq \cdots \leq T_{\left(V_{1}\right)}^{1},
$$

where $T^{1}$ is the first coordinate of $\underline{T}$ and analogously for other columns. Since all points are in the same column, we have for $i=1, \ldots, V_{1}$

$\operatorname{Var}\left(Y\left(\underline{T}_{(i)}\right) \mid Y\left(\underline{T}_{(i-1)}\right)\right) \leq\left(\underline{T}_{(i)}-\underline{T}_{(i-1)}\right)^{2 H} \leq\left[\left(T_{(i)}^{1}-T_{(i-1)}^{1}\right)+(N-1) n^{-1 / N}\right]^{2 H}$. If $\left(U_{i}\right)_{i=0}^{V_{1}}$ are iid $\exp (\lambda)$ then $\left(T_{(i)}^{1}-T_{(i-1)}^{1}\right)_{i=1}^{V_{1}}=^{D}\left(\frac{U_{i}}{\sum_{j=0}^{V_{1}} U_{j}}\right)_{i=1}^{V_{1}}$, see Ross (1996), and the expectation in (2.11) is greater than

$$
\begin{aligned}
\mathbb{E}\left[\prod_{i=1}^{V_{1}}\left(\left(T_{(i)}^{1}-T_{(i-1)}^{1}\right)+(N-1) n^{-1 / N}\right)^{-d H} \mid \underline{\underline{V}}\right] \\
=\mathbb{E}\left[\prod _ { i = 1 } ^ { V _ { 1 } } \left(\frac{U_{i}}{\left.\left.\sum_{j=0}^{V_{1} U_{j}}+\frac{N-1}{n^{1 / N}}\right)^{-d H}\right]}\right.\right. \\
\geq \mathbb{E}\left[\prod_{i=1}^{V_{1}}\left(\frac{2 U_{i}+(N-1)}{n^{1 / N}}\right)^{-d H} I_{\sum_{j=0}^{V_{1}} U_{j} \geq \frac{n^{1 / N}}{2}}\right] .
\end{aligned}
$$

We can write $\prod_{i=1}^{V_{1}}\left(2 U_{i}+(N-1)\right)=\exp \left(\sum_{i=1}^{V_{1}} \log \left(2 U_{i}+(N-1)\right)\right)$, where the variables $\log \left(2 U_{i}+(N-1)\right)$ are iid. Let $\mu(\in(0, \infty))$ be the expectation of $\log \left(2 U_{i}+\right.$ 
$(N-1))$, then using the strong law of large numbers when $V_{1} \nearrow \infty$, we have with probability 1

$$
\prod_{i=1}^{V_{1}}\left(2 U_{i}+(N-1)\right) \leq e^{2 \mu V_{1}} \text { and } \sum_{j=0}^{V_{1}} U_{j} \geq \frac{3}{4} V_{1}
$$

Then on the event $A$

$$
\mathbb{E}\left[\prod_{i=1}^{V_{1}}\left(\frac{2 U_{i}+(N-1)}{n^{1 / N}}\right)^{-d H} I_{\sum_{j=0}^{V_{1}} U_{j} \geq \frac{n^{1 / N}}{2}}\right] \geq \frac{1}{2}\left(\frac{e^{2 \mu}}{n^{1 / N}}\right)^{-d H V_{1}}
$$

We have (on $A$ )

$$
\begin{aligned}
\left(n^{1 / N}\right)^{d H V_{1}} e^{-2 \mu d H V_{1}} & \geq\left(n^{1 / N}\right)^{d H\left(n^{1 / N}-n^{1 /(2 N)} \log n\right)} \cdot e^{-2 \mu d H\left(n^{1 / N}+n^{1 /(2 N)} \log n\right)} \\
& \geq\left(n^{1 / N} e^{2 \mu}\right)^{-d H n^{1 /(2 N)} \log n} \cdot\left(\frac{n^{1 / N}}{e^{2 \mu}}\right)^{d H n^{1 / N}}
\end{aligned}
$$

and

$$
\left[\left(\frac{e^{2 \mu}}{n^{1 / N}}\right)^{-d H V_{1}}\right]^{n^{1-1 / N}} \geq\left(n^{1 / N} e^{2 \mu}\right)^{-d H n^{1-1 / 2 N} \cdot \log n} \cdot\left(\frac{n^{1 / N}}{e^{2 \mu}}\right)^{d H n}
$$

Finally, from (2.6), (2.11)-(2.14) and arguing similarly for the other columns, we have

$$
\begin{aligned}
& \mathbb{E}\left[L_{\underline{0}}\left([0,1]^{N}\right)^{n}\right] \\
& \geq(2 \pi)^{n d / 2} \frac{1}{2}\left(\frac{1}{2}\right)^{n^{1-1 / N}}\left(n^{1 / N} e^{2 \mu}\right)^{-d H n^{1-1 /(2 N)} \cdot \log n} \cdot\left(\frac{n^{1 / N}}{e^{2 \mu}}\right)^{d H n} .
\end{aligned}
$$

This implies, when $n \rightarrow \infty$, that there exists a finite constant $C$ such that

$$
\mathbb{E}\left[L_{\underline{0}}\left([0,1]^{N}\right)^{n}\right]^{\frac{1}{n}} \geq C n^{d H / N},
$$

and this completes the proof of Theorem 5 .

Proof of Lemma 1 Let $X=V-n^{1 / N}$ so we can write

$$
X=\sum_{i=1}^{n} I_{i}-n^{1 / N}, \text { where } I_{i} \sim \operatorname{Bernoulli}\left(n^{\frac{1-N}{N}}\right) .
$$


For $a>0$ small,

$$
\begin{aligned}
\mathbb{E}\left[e^{-a X}\right] & =e^{a n^{1 / N}} \mathbb{E}\left[e^{-a \sum I_{i}}\right] \\
& =e^{a n^{1 / N}}\left[e^{-a} n^{\frac{1-N}{N}}+\left(1-n^{\frac{1-N}{N}}\right)\right]^{n} \\
& =e^{a n^{1 / N}}\left[1+\frac{\left(e^{-a}-1\right) n^{\frac{1}{N}}}{n}\right]^{n} \\
& \leq e^{a n^{1 / N}} e^{\left(e^{-a}-1\right) n^{1 / N}} \text { Taylor's development of log } x \text { as } x \text { small } \\
& =e^{\left(e^{-a}+a-1\right) n^{1 / N}} \\
& \leq e^{a^{2} n^{1 / N}} \text { since } e^{-a}+a-1 \leq a^{2}
\end{aligned}
$$

We have for all $a>0$ small,

$$
\begin{aligned}
\mathbb{P}\left(V-n^{1 / N} \leq-n^{1 /(2 N)} x\right) & \leq \frac{\mathbb{E}\left[e^{-a\left(V-n^{1 / N}\right)}\right]}{e^{a\left(n^{1 /(2 N)} x\right)}} \\
& =\frac{e^{a^{2} n^{1 / N}}}{e^{a n^{1 /(2 N)} x}}=e^{n^{1 / N} a^{2}-a n^{1 /(2 N)} x} .
\end{aligned}
$$

We choose $a$ which minimize (2.17), i.e., $a=\frac{x}{2 n^{1 /(2 N)}}$. We find

$$
\mathbb{P}\left(V-n^{1 / N} \leq-n^{1 /(2 N)} x\right) \leq e^{-x^{2} / 4}
$$

\section{Law of the iterated logarithm}

As noted in the introduction, in this section we consider the comparison processes derived from non-interval choices of $I$. The reason for this choice of $I$ is that we will seek a collection of independent comparison processes.

We consider the collection $I_{n}:=I_{n, \beta}=\left[-n^{\beta}, n^{\beta}\right]^{N} \backslash\left[-n^{-\beta}, n^{-\beta}\right]^{N}$. The parameter $\beta$ is for the moment unspecified but will be large. We first show that if $\beta$ is taken large enough and $\underline{X}^{\prime n}$ is the comparison process based on $I=I_{n}$, then for all $n$ large

$$
\begin{aligned}
\mathbb{E}\left[\left(L_{\underline{0}}^{\underline{X}}\left([0,1]^{N}\right)-L_{\underline{\underline{0}}}^{\underline{X}^{\prime n}}\left([0,1]^{N}\right)\right)^{2}\right] & \left.=2 \int_{[0,1]^{N \times[0,1]^{N}}}\left(P_{\underline{s}, \underline{t}}(\underline{0}, \underline{0})-P_{\underline{s}, \underline{t}}^{\prime} \underline{(0,}, \underline{0}\right)\right) d \underline{s} d \underline{t} \\
& \leq \frac{1}{n^{2}}
\end{aligned}
$$

(for more details, see Baraka and Mountford 1997). 
We first take a constant $\alpha>\max \{3 / H, 3 /(N-d H)\}$ and consider

$$
\int_{\substack{[0,1]^{N} \times[0,1]^{N} \\|\underline{t}-\underline{s}|<1 / n^{\alpha}}}\left(P_{\underline{s}, \underline{t}}(\underline{0}, \underline{0})-P_{\underline{s}, \underline{t}}^{\prime}(\underline{0}, \underline{0})\right) d \underline{s} d \underline{t} .
$$

Note that $P_{\underline{s}, \underline{t}}(\underline{0}, \underline{0}) \geq P_{\underline{s}, \underline{t}}^{\prime}(\underline{0}, \underline{0})$ and by the positivity of densities, (3.2) is bounded by

$$
\int_{\substack{[0,1]^{N} \times[0,1]^{N} \\|\underline{t}-\underline{s}|<1 / n^{\alpha}}} P_{\underline{s}, \underline{t}}(\underline{0}, \underline{0}) d \underline{s} d \underline{t}=2 \int_{\substack{[0,1]^{N} \times[0,1]^{N} \\|\underline{t}-\underline{s}|^{N}<1 / n^{\alpha},|\underline{s}|^{<\mid t} \mid}} P_{\underline{s}, \underline{t}}(\underline{0}, \underline{0}) d \underline{s} d \underline{t} .
$$

This latter expression (by the LND property or more simply by direct computation) is bounded by

$$
C \int_{\substack{[0,1]^{N} \times[0,1]^{N} \\|\underline{t}-\underline{s}|<1 / n^{\alpha},|\underline{s}|<|\underline{|t|}|}} \frac{1}{|\underline{s}|^{d H}} \frac{1}{|\underline{t}-\underline{s}|^{d H}} d \underline{s} d \underline{t},
$$

for some universal $C$. (3.4) is easily seen to be bounded by

$$
C \int_{[0,1]^{N}} \frac{1}{|\underline{s}|^{d H}} d \underline{s} \int_{|\underline{t}|<1 / n^{\alpha}} \frac{1}{|\underline{t}|^{d H}} d \underline{t} .
$$

The bound $\alpha>3 /(N-d H)$ ensures that this is less than $C^{\prime} / n^{3}$ for some universal $C^{\prime}$ which is sufficient for our needs. We have in almost identical fashion that

$$
\int_{\substack{[0,1]^{N} \times[0,1]^{N} \\|\underline{t}| \text { or }|\underline{s}|<1 / n^{\alpha}}} P_{\underline{s}, \underline{t}}(\underline{0}, \underline{0}) d \underline{s} d \underline{t} \leq 2 C^{\prime} / n^{3} .
$$

We now turn to bounding the integral over $\left\{(\underline{s}, \underline{t}) \in[0,1]^{N} \times[0,1]^{N}:|\underline{s}|,|\underline{t}|\right.$, $\left.|\underline{s}-\underline{t}| \geq 1 / n^{\alpha}\right\}$. First we have, as in Baraka and Mountford (1997),

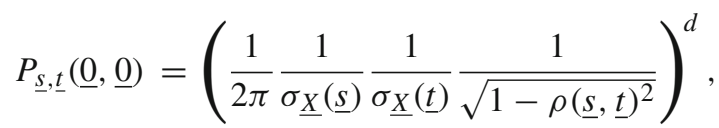

where $\rho(\underline{s}, \underline{t})$ is the correlation between $X_{1}(\underline{s})$ and $X_{1}(\underline{t})$. Since the one dimensional distributions of $\underline{X}$ and $\underline{X}^{\prime n}$ are the same, we have equally

$$
P_{\underline{s}, \underline{t}}^{\prime}(\underline{0}, \underline{0})=\left(\frac{1}{2 \pi} \frac{1}{\sigma_{\underline{X}}(\underline{s})} \frac{1}{\sigma_{\underline{X}}(\underline{t})} \frac{1}{\sqrt{1-\rho^{\prime}(\underline{s}, \underline{t})^{2}}}\right)^{d},
$$


where $\rho^{\prime}(\underline{s}, \underline{t})$ is the correlation between $X_{1}(\underline{s})$ and $X_{1}^{\prime n}(\underline{t})$ [and so between $X_{1}^{\prime n}(\underline{s})$ and $X_{1}(\underline{t})$. It follows from (3.6) and (3.7) that

$$
\begin{aligned}
& \left|P_{\underline{s}, \underline{t}}(\underline{0}, \underline{0})-P_{\underline{s}, \underline{t}}^{\prime}(\underline{0}, \underline{0})\right| \\
& \leq 2^{d-1}\left(\frac{1}{2 \pi} \frac{1}{\sigma_{X}(\underline{s})} \frac{1}{\sigma_{X}(\underline{t})}\left(\frac{1}{\sqrt{1-\rho(\underline{s}, \underline{t})^{2}}}-\frac{1}{\sqrt{1-\rho^{\prime}(\underline{s}, \underline{t})^{2}}}\right)\right)^{d} .
\end{aligned}
$$

Now

$$
\left|\rho(\underline{s}, \underline{t})-\rho^{\prime}(\underline{s}, \underline{t})\right|=\left|\frac{\mathbb{E}\left(X_{1}(\underline{t}) X_{1}(\underline{s})\right)-\mathbb{E}\left(X_{1}(\underline{t}) X_{1}^{\prime}(\underline{s})\right)}{\sigma_{X}(\underline{s}) \sigma_{X}(\underline{t})}\right| .
$$

Similar to (1.11), the numerator of (3.9) is equal to

$$
\begin{aligned}
& C_{N, H}^{2} \int_{I_{n}^{c}}\left(\frac{1}{|\underline{x}-\underline{t}|^{N / 2-H}}-\frac{1}{|\underline{x}|^{N / 2-H}}\right)\left(\frac{1}{|\underline{x}-\underline{s}|^{N / 2-H}}-\frac{1}{|\underline{x}|^{N / 2-H}}\right) d \underline{x} \\
& =C_{N, H}^{2} \int_{\left[-n^{-\beta}, n^{-\beta}\right]^{N}}\left(\frac{1}{|\underline{x}-\underline{t}|^{N / 2-H}}-\frac{1}{|\underline{x}|^{N / 2-H}}\right)\left(\frac{1}{|\underline{x}-\underline{s}|^{N / 2-H}}-\frac{1}{|\underline{x}|^{N / 2-H}}\right) d \underline{x} \\
& \quad+C_{N, H}^{2} \int_{\mathbb{R}^{N} \backslash\left[-n^{\beta}, n^{\beta}\right]^{N}}\left(\frac{1}{|\underline{x}-\underline{t}|^{N / 2-H}}-\frac{1}{|\underline{x}|^{N / 2-H}}\right)\left(\frac{1}{|\underline{x}-\underline{s}|^{N / 2-H}}-\frac{1}{|\underline{x}|^{N / 2-H}}\right) d \underline{x} .
\end{aligned}
$$

Recall that we are interested in $(\underline{s}, \underline{t})$ pairs such that $\left\{(\underline{s}, \underline{t}):|\underline{s}|,|\underline{t}|,|\underline{s}-\underline{t}| \geq 1 / n^{\alpha}\right\}$. For the first integral we have the simple bound (given $\alpha<\beta$ and $n$ large)

$$
C_{N, H}^{2} \int_{\left[-n^{-\beta}, n^{-\beta}\right]^{N}} \frac{1}{|\underline{x}|^{N-2 H}} d \underline{x} \leq C^{\prime \prime} / n^{(2 H) \beta} .
$$

For the second integral we use (1.11) to obtain similarly the bound $C^{\prime \prime} / n^{(2-2 H) \beta}$. Note that if $\underline{s}$ and $\underline{t}$ are both $\geq n^{-\alpha}$, then $\sigma_{\underline{X}}(\underline{s})$ and $\sigma_{\underline{X}}(\underline{t})$ are both bounded below by $1 / n^{\alpha H}$. This and (3.11) together yield the bound

$$
\left|\rho(\underline{s}, \underline{t})-\rho^{\prime}(\underline{s}, \underline{t})\right| \leq C_{1}\left(n^{-2(\beta-\alpha) H}+n^{-2(\beta(1-H)-\alpha H)}\right) .
$$

Furthermore if $|\underline{s}-\underline{t}| \geq n^{-\alpha}$, then we have for a universal constant $C_{2}$ that $\sqrt{1-\rho(\underline{s}, \underline{t})^{2}} \geq C_{2} n^{-\alpha H}>>\left|\rho(\underline{s}, \underline{t})-\rho^{\prime}(\underline{s}, \underline{t})\right|$.

It only remains to apply elementary calculus and (3.12) to see that

$$
\left|\frac{1}{\sqrt{1-\rho(\underline{s}, \underline{t})^{2}}}-\frac{1}{\sqrt{1-\rho^{\prime}(\underline{s}, \underline{t})^{2}}}\right| \leq \frac{C_{3}}{n^{-3 \alpha H}}\left(n^{-2(\beta-\alpha) H}+n^{-2(\beta(1-H)-\alpha H)}\right),
$$

which will be less than $\frac{1}{n^{3}}$ for $n$ large provided $\beta$ was chosen sufficiently large. 
It is now clear that (3.1) follows from (3.5), (3.8) and (3.13). Consequently we have

$$
\mathbb{P}\left(\left|L_{\underline{\underline{X}}}^{\underline{X}}\left([0,1]^{N}\right)-L_{\underline{\underline{0}}}^{\underline{X}^{\prime}}\left([0,1]^{N}\right)\right| \geq 1\right) \leq \frac{C}{n^{2}}
$$

for some $C<\infty$. By scaling we immediately have

$$
\mathbb{P}\left(\left|L_{\underline{\underline{X}}}^{\underline{X}}\left([0, h]^{N}\right)-L_{\underline{\underline{0}}}^{\underline{X}^{\prime}}\left([0, h]^{N}\right)\right| \geq h^{N-H d}\right) \leq \frac{C}{n^{2}} .
$$

for $\underline{X}^{\prime}$ the comparison process derived from $I_{n}$.

Applying the first Borel-Cantelli lemma to the events

$$
A_{n}=\left\{\left|L_{\underline{\underline{0}}}^{\underline{X}}\left(\left[0,2^{-n}\right]^{N}\right)-L_{\underline{0}}^{\underline{X}^{\prime}}\left(\left[0,2^{-n}\right]^{N}\right)\right| \geq 2^{-n(N-H d)}\right\}
$$

we have

$$
\mathbb{P}\left(\limsup _{n \rightarrow \infty} A_{n}\right)=0 \text {. }
$$

Now choose a sequence $1 \ll n_{0}<n_{1}<\cdots$ as follows. Given $n_{i}$ let $n_{i+1}$ be the smallest positive integer so that

$$
2^{-n_{i+1}}\left(n_{i+1}\right)^{\beta}<\frac{2^{-n_{i}}}{n_{i}^{\beta}}
$$

We have easily $\frac{n_{i+1}-n_{i}}{\log _{2} n_{i}} \rightarrow 2 \beta$.

The point is that the comparison processes $\underline{X}_{i}^{\prime}$ derived from $I_{n_{i}}$ are (or more accurately may be taken to be) independent.

By (2.1) we can choose a constant $h$, depending on $N, H$, and $d$ only, such that

$$
B_{i}=\left\{L_{\underline{0}}^{\underline{X}_{i}^{\prime}}\left(\left[0,2^{-n_{i}}\right]^{N}\right) \geq h 2^{-n_{i}(N-H d)}\left(\log n_{i}\right)^{\frac{H d}{N}}\right\}
$$

has probability $\geq \frac{C}{\sqrt{n_{i}}}$.

And so given our bounds on $n_{i+1}-n_{i}$

$$
\sum \mathbb{P}\left(B_{i}\right)=\infty
$$

By the independence of the processes $\underline{X}_{i}^{\prime}$ and the second Borel-Cantelli lemma, we have

$$
\mathbb{P}\left(\limsup _{i \uparrow \infty} B_{i}\right)=1 .
$$


Given (3.16) we conclude that almost surely

$$
\limsup _{n \rightarrow \infty}\left\{L \underline{\underline{0}}\left(\left[0,2^{-n_{i}}\right]^{N}\right) \geq h 2^{-n_{i}(N-H d)}\left(\left(\log n_{i}\right)^{\frac{H d}{N}}-1\right)\right\}
$$

occurs. That is

$$
\underset{r \downarrow 0}{\lim \sup } \frac{L_{\underline{0}}^{\underline{X}}\left([0, r]^{N}\right)}{r^{N-H d}\left(\log \log \frac{1}{r}\right)^{\frac{H d}{N}}} \geq h
$$

Now let us fix $g$ and let $\underline{X}$ be the comparison process derived from $I=[-g, g]^{N}$. It is easy to establish that

$$
\underset{r \downarrow 0}{\limsup } \frac{L_{\underline{\underline{x}}}^{\underline{X}}\left([0, r]^{N}\right)-L_{\underline{\underline{0}}}^{\underline{X}^{\prime}}\left([0, r]^{N}\right)}{r^{N-H d}\left(\log \log \frac{1}{r}\right)^{\frac{H d}{N}}}=0 \quad \text { almost surely. }
$$

That is, the r.v. $\lim \sup _{r \downarrow 0} \frac{L_{\underline{0}}^{\underline{X}}\left([0, r]^{N}\right)}{r^{N-H d}\left(\log \log \frac{1}{r}\right)^{\frac{H d}{N}}}$ is independent of $\sigma\left(\dot{W}(\underline{x}), \underline{x} \in I^{c}\right)$.

The arbitrariness of $g$ and the zero-one law in Pitt and Tran (1979) give the triviality of

$$
\underset{r \downarrow 0}{\lim \sup } \frac{L \underline{\underline{X}}\left([0, r]^{N}\right)}{r^{N-H d}\left(\log \log \frac{1}{r}\right)^{\frac{H d}{N}}}
$$

and we are done.

Remark that with a little more work, we can identify the constant $C_{1.6}$.

\section{The non-strong LND property for process $\underline{U}$}

Let $\underline{U}=\left\{\underline{U}(\underline{t}): \underline{t} \in[-b, b]^{N}\right\}$ a process with values in $\mathbb{R}^{d}$ defined by

$$
U_{i}(\underline{t})=C_{N, H} \int_{[-a, a]^{N}} f(\underline{x}, \underline{t}) d \dot{W}_{i}(\underline{x})
$$

where $f(\underline{x}, \underline{t})=\frac{1}{|\underline{x}-\underline{t}|^{N / 2-H}}-\frac{1}{|\underline{x}|^{N / 2-H}}, \underline{\dot{W}}$ is a $d$-dimensional white noise on $\mathbb{R}^{N}$ and $a>>b$.

Proposition 2 For any integer $r \geq 2$, there exists a positive constant $C_{r}>0$ such that for any $\underline{t}_{1}, \ldots, \underline{t}_{r} \in[-b, b]^{N}$ we have 


$$
\operatorname{Var}\left(\underline{U}\left(\underline{t}_{1}\right) \mid \underline{U}\left(\underline{t}_{i}\right), 2 \leq i \leq r\right) \geq C_{r} h^{2 H},
$$

where $h=\min \left\{\left|\underline{t}_{1}\right| ;\left|\underline{t}_{1}-\underline{t}_{i}\right|, i \geq 2\right\}$.

Proof In fact (4.2) is equivalent to prove that for $r \geq 1$, there exists a constant $C_{r}>0$ such that for any reals $a_{1}, \ldots, a_{r} \in \mathbb{R}$ and any $\underline{t}_{1}, \ldots, \underline{t}_{r} \in[-b, b]^{N}$, we have

$$
\mathbb{E}\left[\left(\underline{U}\left(\underline{t}_{1}\right)-\sum_{i=2}^{r} a_{i} \underline{U}\left(\underline{t}_{i}\right)\right)^{2}\right] \geq C_{r} h^{2 H},
$$

where $h=\min \left\{\left|\underline{t}_{1}\right| ;\left|\underline{t}_{1}-\underline{t}_{i}\right|, i \geq 2\right\}$.

Since that it is evident for $r=1$, we will arguing inductively (although the inductive hypothesis will only be used for case 2 below) and suppose that there exists a constant $C_{r-1}>0$ such that for any reals $a_{1}, \ldots, a_{r-1} \in \mathbb{R}$ and any $\underline{t}_{1}, \ldots, \underline{t}_{r-1} \in[-b, b]^{N}$, we have

$$
\mathbb{E}\left[\left(\underline{U}\left(\underline{t}_{1}\right)-\sum_{i=2}^{r-1} a_{i} \underline{U}\left(\underline{t}_{i}\right)\right)^{2}\right] \geq C_{r-1} h^{\prime 2 H},
$$

where $h^{\prime}=\min \left\{\left|\underline{t}_{1}\right| ;\left|\underline{t}_{1}-\underline{t}_{i}\right|, r-1 \geq i \geq 2\right\}$. We distinguish two cases.

1. If $\max _{2 \leq i \leq r}\left|a_{i}\right| \leq 1$. Let $I_{r}=\left\{\underline{x}:\left|\underline{x}-\underline{t}_{1}\right| \leq h / k\right\}$, where $k$ is large enough and will be specified in each case.

(a) If $N / 2-H>0$ : for $\underline{x} \in I_{r}$,

$$
|\underline{x}| \geq\left(1-\frac{1}{k}\right) h \geq(k-1)\left|\underline{x}-\underline{t}_{1}\right| \text { and }\left|\underline{x}-\underline{t}_{i}\right| \geq(k-1)\left|\underline{x}-\underline{t}_{1}\right| ; i \geq 2
$$

this implies that

$$
f\left(\underline{t}_{1}, \underline{x}\right)=\frac{1}{\left|\underline{x}-\underline{t}_{1}\right|^{N / 2-H}}-\frac{1}{|\underline{x}|^{N / 2-H}} \geq\left(1-\frac{1}{(k-1)^{N / 2-H}}\right) \frac{1}{\left|\underline{x}-\underline{t}_{1}\right|^{N / 2-H}}
$$

and for each $i \geq 2$

$$
\left|f\left(\underline{t}_{i}, \underline{x}\right)\right|=\left|\frac{1}{|\underline{x}-\underline{t}|^{N / 2-H}}-\frac{1}{|\underline{x}|^{N / 2-H}}\right| \leq \frac{2}{(k-1)^{N / 2-H}} \frac{1}{\left|\underline{x}-\underline{t}_{1}\right|^{N / 2-H}} .
$$


So

$$
\begin{aligned}
\mathbb{E}\left[\left(\underline{U}\left(\underline{t}_{1}\right)-\sum_{i=2}^{r} a_{i} \underline{U}\left(\underline{t}_{i}\right)\right)^{2}\right] & =\int_{[-a, a]^{N}}\left(f\left(\underline{t}_{1}, \underline{x}\right)-\sum_{i=2}^{r} a_{i} f\left(\underline{t}_{i}, \underline{x}\right)\right)^{2} d \underline{x} \\
& \geq \int_{I_{r}}\left(f\left(\underline{t}_{1}, \underline{x}\right)-\sum_{i=2}^{r} a_{i} f\left(\underline{t}_{i}, \underline{x}\right)\right)^{2} d \underline{x} \\
\text { If } \frac{2 r}{(k-1)^{N / 2-H}}<1: & \geq\left(1-\frac{2 r}{(k-1)^{N / 2-H}}\right)^{2} \int_{I_{r}} \frac{1}{\left|\underline{x}-\underline{t}_{1}\right|^{N-2 H}} d \underline{x} \\
& \geq C_{r .1} h^{2 H} .
\end{aligned}
$$

In this case, it is sufficient to take $k$ such that $(k-1)^{N / 2-H}>2 r$ and $C_{r .1}$ is a constant which depend only on $r$.

(b) If $N / 2-H<0$ : this implies that $N=1$ and $H>1 / 2$. Let

$$
g(x)=|x|^{H-1 / 2}+\sum_{i=2}^{r} a_{i}\left(\left|x-t_{i}\right|^{H-1 / 2}-|x|^{H-1 / 2}\right) .
$$

We have for $\left|x-t_{1}\right| \leq \frac{h}{2}$

$$
\begin{aligned}
\left|g^{\prime}(x)\right| & \leq(H-1 / 2)\left[|x|^{H-3 / 2}+\sum_{i=2}^{r}\left|a_{i}\right|\left(\left|x-t_{i}\right|^{H-3 / 2}+|x|^{H-3 / 2}\right)\right] \\
& \leq(H-1 / 2)\left(\frac{h}{2}\right)^{H-3 / 2}\left(1+2 \sum_{i=2}^{r}\left|a_{i}\right|\right) \\
& \leq 2 r(H-1 / 2)\left(\frac{h}{2}\right)^{H-3 / 2} .
\end{aligned}
$$

Let $J_{r}=\left\{x: \frac{2 h}{k} \leq\left|x-t_{1}\right| \leq \frac{3 h}{k}\right\}$. Remark that for $x \in J_{r}$ and $y \in I_{r}$, we have

$$
\begin{aligned}
\left|x-t_{1}\right|-\left|y-t_{1}\right| & \geq\left(\frac{2 h}{k}\right)^{H-1 / 2}-\left(\frac{h}{k}\right)^{H-1 / 2} \\
& =\left(2^{H-1 / 2}-1\right)\left(\frac{h}{k}\right)^{H-1 / 2}=C_{4.1} h^{H-1 / 2} .
\end{aligned}
$$

and

$$
|g(x)-g(y)| \leq \max _{z \in I_{r} \cup J_{r}}\left|g^{\prime}(z)\right||x-y| \leq 2 r(H-1 / 2)\left(\frac{h}{2}\right)^{H-3 / 2} \cdot \frac{4 h}{k} .
$$


We take $k$ such that $2 r(H-1 / 2)\left(\frac{1}{2}\right)^{H-3 / 2} \cdot \frac{4}{k} \leq \frac{C_{4.1}}{4}$, this implies that

$$
k^{3 / 2-H} \geq \frac{8 r(H-1 / 2)}{2^{H-3 / 2}\left(2^{H-1 / 2}-1\right)} .
$$

Suppose that ||$x-\left.t_{1}\right|^{H-1 / 2}-g(x) \mid \geq \frac{C_{4.1}}{4} h^{H-1 / 2}$, so for all $x \in I_{r}$

$$
\begin{aligned}
\mathbb{E}\left[\left(\underline{U}\left(t_{1}\right)-\sum_{i=2}^{r} a_{i} \underline{U}\left(t_{i}\right)\right)^{2}\right] & \geq \int_{I_{r}}\left(\left|x-t_{1}\right|^{H-1 / 2}-g(x)\right)^{2} d x \\
& \geq \frac{C_{4.1}^{2}}{16} h^{2 H-1} \times \frac{2 h}{k}=C_{r .2} h^{2 H} .
\end{aligned}
$$

If not, there exists $y \in I_{r}$ such that ||$y-\left.t_{1}\right|^{H-1 / 2}-g(y) \mid<\frac{C_{4.1}}{4} h^{H-1 / 2}$. But for $x \in J_{r}$ we have

$$
\begin{aligned}
|| x-\left.t_{1}\right|^{H-1 / 2}-g(x) \mid \geq & \left|x-t_{1}\right|^{H-1 / 2}-\left|y-t_{1}\right|^{H-1 / 2}-|g(x)-g(y)| \\
& -|| y-\left.t_{1}\right|^{H-1 / 2}-g(y) \mid \\
\geq & C_{4.1} h^{H-1 / 2}-\frac{C_{4.1}}{4} h^{H-1 / 2}-\frac{C_{4.1}}{4} h^{H-1 / 2} \\
= & \frac{C_{4.1}}{2} h^{H-1 / 2} .
\end{aligned}
$$

Since

$$
\int\left(\left|x-t_{1}\right|^{H-1 / 2}-g(x)\right)^{2} d x \geq \int_{J_{r}}\left(\left|x-t_{1}\right|^{H-1 / 2}-g(x)\right)^{2} d x
$$

we conclude that

$$
\mathbb{E}\left[\left(\underline{U}\left(t_{1}\right)-\sum_{i=2}^{r} a_{i} \underline{U}\left(t_{i}\right)\right)^{2}\right] \geq C_{r .3} h^{2 H}
$$

(c) If $N / 2-H=0$ : this implies that $N=1$ and $H=1 / 2$ and we have classical Brownian motion.

2. If $\max _{2 \leq i \leq r}\left|a_{i}\right|>1$ : without loss of generality, we take $a_{r}=\max _{2 \leq i \leq r} a_{i}$. Let $h_{2}=\min \left\{\left|\underline{t}_{r}\right| ;\left|\underline{t}_{r}-\underline{t}_{i}\right|, i \leq r\right\}$ and $C_{r .4}=\min \left(C_{r .1}, C_{r .2}, C_{r .3}\right)$. 
a. If $\left|a_{r}\right| h_{2}^{H} \geq \sqrt{\delta} h^{H}$ where $\delta=\min \left(C_{r .4}, C_{r-1}\right) / 2$,

$$
\begin{aligned}
& \mathbb{E}\left[\left(\underline{U}\left(\underline{t}_{1}\right)-\sum_{i=2}^{r} a_{i} \underline{U}\left(\underline{t}_{i}\right)\right)^{2}\right] \\
& \left.=\left|a_{r}\right|^{2} \mathbb{E}\left[\left(\underline{U}\left(\underline{t}_{r}\right)-\sum_{i=1}^{r-1} \frac{a_{i}}{a_{r}} \underline{U}\left(\underline{t}_{i}\right)\right)^{2}\right] \quad \text { (where } a_{1}=-1\right) \\
& \geq\left|a_{r}\right|^{2} C_{r .4} h_{2}^{2 H} \geq C_{r .5} h^{2 H} .
\end{aligned}
$$

b. If $\left|a_{r}\right| h_{2}^{H}<\sqrt{\delta} h^{H}$, i.e., is small w.r.t. $h$. We will proceed inductively.

$$
\begin{aligned}
\mathbb{E} & {\left[\left(\underline{U}\left(\underline{t}_{1}\right)-\sum_{i=2}^{r} a_{i} \underline{U}\left(\underline{t}_{i}\right)\right)^{2}\right]^{1 / 2} } \\
= & \mathbb{E}\left[\left(\underline{U}\left(\underline{t}_{1}\right)-\sum_{i=2}^{r-1} a_{i}^{\prime} \underline{U}\left(\underline{t}_{i}\right)-a_{r}\left(\underline{U}\left(\underline{t}_{r}\right)-\underline{U}\left(\underline{t}_{r-1}\right)\right)\right)^{2}\right]^{1 / 2} \\
\geq & \left\{\mathbb{E}\left[\left(\underline{U}\left(\underline{t}_{1}\right)-\sum_{i=2}^{r-1} a_{i}^{\prime} \underline{U}\left(\underline{t}_{i}\right)\right)^{2}\right]\right\}^{1 / 2} \\
& -\left|a_{r}\right|\left\{\mathbb{E}\left[\left(\underline{U}\left(\underline{t}_{r}\right)-\underline{U}\left(\underline{t}_{r-1}\right)\right)^{2}\right]\right\}^{1 / 2} \\
\geq & \sqrt{C_{r-1}} h^{H}-\left|a_{r}\right| h_{2}^{H} \geq \sqrt{C_{r .6}} h^{H} .
\end{aligned}
$$

We conclude by taking $C_{r}=\min \left(C_{r .4}, C_{r .5}, C_{r .6}\right)$.

\section{A bound on local times differences}

In this section we achieve a better bound for the difference between the local time for our original process and that for a comparison process.

We let $\underline{Z}$ be an independent white noise and we fix a parameter $h>0, h$ will be small so $h^{\overline{1-\epsilon}} \gg h$.

We write

$$
\underline{X}^{\prime}(\underline{t})=C_{N, H} \int_{\mathbb{R}^{N}}\left(\frac{1}{|\underline{x}-\underline{t}|^{\frac{N}{2}-H}}-\frac{1}{|\underline{x}|^{\frac{N}{2}-H}}\right) d \underline{\dot{W}^{\prime}}(\underline{x})
$$

for

$$
\begin{aligned}
\dot{W}^{\prime}(\underline{x}) & =\underline{\dot{W}}(\underline{x}) & & \underline{x} \in\left[-h^{1-\epsilon}, h^{1-\epsilon}\right]^{N} \\
& =\underline{\dot{Z}}(\underline{x}) & & \underline{x} \notin\left[-h^{1-\epsilon}, h^{1-\epsilon}\right]^{N} .
\end{aligned}
$$


Then we have

$$
\begin{aligned}
& \underline{X}(\underline{t})=\underline{U}(\underline{t})+\underline{V}(\underline{t}) \\
& \underline{X^{\prime}}(\underline{t})=\underline{U}(\underline{t})+\underline{V^{\prime}}(\underline{t})
\end{aligned}
$$

for

$$
\begin{aligned}
\underline{U}(\underline{t}) & =C_{N, H} \int_{\underline{x} \in\left[-h^{1-\epsilon}, h^{1-\epsilon}\right]^{N}}\left(\frac{1}{|\underline{x}-\underline{t}|^{\frac{N}{2}-H}}-\frac{1}{|\underline{x}|^{\frac{N}{2}-H}}\right) d \underline{\dot{W}^{\prime}}(\underline{x}) \\
& =C_{N, H} \int_{\underline{x} \in\left[-h^{1-\epsilon}, h^{1-\epsilon}\right]^{N}}\left(\frac{1}{|\underline{x}-\underline{t}|^{\frac{N}{2}-H}}-\frac{1}{|\underline{x}|^{\frac{N}{2}-H}}\right) d \underline{\dot{W}}(\underline{x}) .
\end{aligned}
$$

Then by design $\underline{U}, \underline{V}, \underline{V^{\prime}}$ are independent processes.

We will study $L_{\underline{\underline{x}}}^{\underline{X}}\left([-h, h]^{N}\right)-L_{\underline{\underline{X}}}\left([-h, h]^{N}\right)$. The first point to be made is

Lemma 2 For $\epsilon>0$ and $h$ small, there exists a constant $c>0$ such that

$$
\mathbb{P}\left(\sup _{\underline{t} \in[-h, h]^{N}}|\underline{V}(\underline{t})| \geq h^{H} h^{(2-H) \frac{\epsilon}{3}}\right) \leq K e^{-c h^{-(2-H) \frac{\epsilon}{3}}}
$$

This lemma follows from a routine application of the Gaussian isoperimetric inequality, see, e.g., Talagrand (1995, Lemma 2.1).

Remark Obviously this bound holds also for process $\underline{V^{\prime}}(\underline{t}), \underline{t} \in[-h, h]^{N}$.

The significance is that the bound $h^{H} h^{(2-H) \frac{\epsilon}{3}}$ (for $h$ small) is very small compared to $h^{H}$ the "typical" size of $|\underline{X}(\underline{t})|$ for $\underline{t} \in[-h, h]^{N}$.

Let $A$ be the event

$$
\left\{\sup _{\underline{t} \in[-h, h]^{N}}|\underline{V}(\underline{t})|, \sup _{\underline{t} \in[-h, h]^{N}}\left|\underline{V^{\prime}}(t)\right| \leq h^{H} h^{(2-H) \frac{\epsilon}{3}}\right\} .
$$

We consider (see, e.g., Baraka and Mountford 1997)

$$
\begin{aligned}
\mathbb{E} & {\left[\left(L_{\underline{\underline{x}}}^{\underline{X}}\left([-h, h]^{N}\right)-L_{\underline{\underline{\underline{X}}}}^{\underline{X^{\prime}}}\left([-h, h]^{N}\right)\right)^{2 k} \mid A\right] } \\
& =\mathbb{E}\left[\int_{\left(\mathbb{R}^{d}\right)^{2 k}} d \underline{z}_{1} \cdots d \underline{z}_{2 k} \int_{\left([-h, h]^{N}\right)^{2 k}} \prod_{j=1}^{2 k}\left(e^{i \underline{z}_{j} \cdot \underline{X}\left(\underline{t}_{j}\right)}-e^{i \underline{z}_{j} \cdot \underline{X}^{\prime}\left(\underline{t}_{j}\right)}\right) d \underline{t}_{1} \cdots d \underline{t}_{2 k} \mid A\right]
\end{aligned}
$$




$$
\begin{aligned}
= & \mathbb{E}\left[\int_{\left(\mathbb{R}^{d}\right)^{2 k}} d \underline{z}_{1} \cdots d \underline{z}_{2 k} \int_{\left([-h, h]^{N}\right)^{2 k}} e^{i \sum_{j=1}^{2 k} \underline{z}_{j} \cdot \underline{U}\left(\underline{t}_{j}\right)}\right. \\
& \left.\times \prod_{j=1}^{2 k}\left[e^{\left.i \underline{z}_{j} \cdot \underline{V} \underline{t}_{j}\right)}-e^{i \underline{z}_{j} \cdot \underline{V}^{\prime}\left(\underline{t}_{j}\right)}\right] d \underline{t}_{1} \cdots d \underline{t}_{2 k} \mid A\right] .
\end{aligned}
$$

As in Geman and Horowitz (1980), Geman et al. (1984) and Xiao (1997) we now use the inequality

$$
\left|e^{i \underline{x}}-e^{i \underline{y}}\right| \leq 2^{\gamma-1}|\underline{x}-\underline{y}|^{\gamma}
$$

for $0<\gamma<1$ to derive that, on the event $A$,

$$
\prod_{j=1}^{2 k}\left|e^{i \underline{z}_{j} \cdot \underline{V}\left(\underline{t}_{j}\right)}-e^{i \underline{z}_{j} \cdot \underline{V}^{\prime}\left(\underline{t}_{j}\right)}\right| \leq\left(\prod_{j=1}^{2 k}\left|\underline{z}_{j}\right|^{\gamma}\right) 2^{2 k \gamma} h^{2 k \gamma H} h^{(2-H) \frac{\epsilon}{3} \gamma 2 k}
$$

So, and here it is crucial that $\underline{U}$ is independent of $\underline{V}$ and $\underline{V^{\prime}}$,

$$
\begin{aligned}
\mathbb{E} & {\left[\left(L_{\underline{0}}^{\underline{X}}\left([-h, h]^{N}\right)-L_{\underline{0}^{\prime}}^{\underline{X}^{\prime}}\left([-h, h]^{N}\right)\right)^{2 k} \mid A\right] } \\
= & 2^{2 k \gamma} \int_{\left(\mathbb{R}^{d}\right)^{2 k}} \prod_{j=1}^{2 k} d \underline{z}_{j} \int_{\left([-h, h]^{N}\right)^{2 k}} \mathbb{E}\left[\prod_{j=1}^{2 k}\left(e^{i \underline{z}_{j} \cdot \underline{X}\left(\underline{t}_{j}\right)}-e^{i \underline{z}_{j} \cdot \underline{X}^{\prime}\left(\underline{t}_{j}\right)}\right) \mid A\right] \prod_{j=1}^{2 k} d \underline{t}_{j} \\
\leq & 2^{2 k \gamma} \int_{\left(\mathbb{R}^{d}\right)^{2 k}} \prod_{j=1}^{2 k} d \underline{z}_{j} \int_{\left([-h, h]^{N}\right)^{2 k}} e^{-\frac{1}{2} \operatorname{Var}\left(\sum_{1}^{2 k} \underline{z}_{j} \cdot \underline{U}\left(\underline{t}_{j}\right)\right)} \\
& \times\left.\prod_{j=1}^{2 k} \underline{z}_{j}\right|^{\gamma} h^{2 k \gamma H} h^{(2-H) \frac{\epsilon}{3} 2 \gamma k} \prod_{j=1}^{2 k} d \underline{t}_{j} \\
= & 2^{2 k \gamma} h^{2 k \gamma H} h^{(2-H) \frac{\epsilon}{3} 2 \gamma k} \int_{\left(\mathbb{R}^{d}\right)^{2 k}} \prod_{j=1}^{2 k} \mid \underline{z}_{j} \gamma^{\gamma} d \underline{z}_{j} \int_{\left([-h, h]^{N}\right)^{2 k}} e^{-\frac{1}{2} \operatorname{Var}\left(\sum_{1}^{2 k} \underline{z}_{j} \cdot \underline{U}\left(\underline{t}_{j}\right)\right)} \prod_{j=1}^{2 k} d \underline{t}_{j} .
\end{aligned}
$$

We emphasize that for the moment $\gamma$ is not fixed.

It is here that we use the (non-strong) LND property of the process $\{\underline{U}(\underline{t}): \underline{t} \in$ $\left.[-h, h]^{N}\right\}$, shown in Proposition 2 to obtain the bound

$$
\operatorname{Var}\left(\sum_{j=1}^{2 k} \underline{z}_{j} \cdot \underline{U}\left(\underline{t}_{j}\right)\right) \geq C_{k} \sum_{1}^{2 k}\left|\underline{z}_{j}\right|^{2}\left|\underline{t}_{j}^{*}\right|^{2 H},
$$


where $\left|\underline{t}_{j}^{*}\right|=\min _{i \neq j}\left\{\left|\underline{t}_{j}\right|,\left|\underline{t}_{j}-\underline{t}_{i}\right|\right\}$. So

$$
\begin{aligned}
\int_{\left(\mathbb{R}^{d}\right)^{2 k}} e^{-\frac{1}{2} \operatorname{Var} \sum_{1}^{2 k} \underline{z}_{j} \cdot \underline{U}\left(\underline{t}_{j}\right)} \prod_{j=1}^{2 k}\left|\underline{z}_{j}\right|^{\gamma} d \underline{z}_{j} & \leq \int_{\left(\mathbb{R}^{d}\right)^{2 k}} e^{-\frac{1}{2} C_{n} \sum\left|\underline{z}_{j}\right|^{2}\left(\underline{t}_{j}^{*}\right)^{2 H}} \prod_{j=1}^{2 k}\left|\underline{z}_{j}\right|^{\gamma} d \underline{z}_{j} \\
& =\prod_{j=1}^{2 k} \int_{\mathbb{R}^{d}}\left|\underline{z}_{j}\right|^{\gamma} e^{-\frac{1}{2} C_{n}\left|\underline{z}_{j}\right|^{2}\left|\underline{t}_{j}^{*}\right|^{2 H}} d \underline{z}_{j} \\
& =C_{k, \gamma} \prod_{j=1}^{2 k} \frac{1}{\left|\underline{t}_{j}^{*}\right|^{H(d+\gamma)}},
\end{aligned}
$$

for a universal constant $C_{k, \gamma}$.

Putting this in the above expression yields

$$
\begin{aligned}
& \mathbb{E}\left[\left(L_{\underline{0}}^{\underline{X}}\left([-h, h]^{N}\right)-L_{\underline{0}}^{\frac{X^{\prime}}{}}\left([-h, h]^{N}\right)\right)^{2 k} \mid A\right] \\
& \leq h^{2 k \gamma H} h^{(2-H) \frac{\epsilon}{3} 2 \gamma k} \int_{\left([-h, h]^{N}\right)^{2 k}} \prod_{j=1}^{2 k} \frac{d \underline{t}_{j}}{\left|\underline{t}_{j}^{*}\right|^{H(d+\gamma)}} .
\end{aligned}
$$

Now it follows easily that

$$
\int_{\left([-h, h]^{N}\right)^{2 k}} \prod_{j=1}^{2 k} \frac{d \underline{t}_{j}}{\left|\underline{t}_{j}^{*}\right|^{H(d+\gamma)}}
$$

is bounded by $F_{k}\left(h^{N-H(d+\gamma)}\right)^{2 k}$ and so for some universal $C_{k, \gamma}^{\prime}$ we have

$$
\mathbb{E}\left[\left(L_{\underline{0}}^{\underline{X}}\left([-h, h]^{N}\right)-L_{\underline{0}}^{\underline{X}^{\prime}}\left([-h, h]^{N}\right)\right)^{2 k} \mid A\right] \leq C_{k, \gamma}^{\prime}\left(h^{N-d H}\right)^{2 k} h^{(2-H) \frac{\epsilon}{3} 2 \gamma k}
$$

We may take $\gamma$ to be any value between 0 and 1 so we have shown

Proposition 3 For $X$ and $X^{\prime}$ as above and any integer $k$ we have

$$
\mathbb{E}\left[\left(L_{\underline{0}}^{\underline{X}}\left([-h, h]^{N}\right)-L_{\underline{0}}^{\underline{X}^{\prime}}\left([-h, h]^{N}\right)\right)^{2 k} \mid A\right] \leq C_{k, \gamma}^{\prime}\left(h^{N-d H}\right)^{2 k} h^{\frac{\epsilon}{3} 2 k} .
$$

Corollary 1 For $\epsilon \in(0,1)$, there exists a constant $C_{\epsilon}$ so that for $h$ sufficiently small

$$
\mathbb{P}\left(\left|L \underline{\underline{X}}\left([-h, h]^{N}\right)-L_{\underline{0}}^{\underline{X}^{\prime}}\left([-h, h]^{N}\right)\right| \geq h^{N-d H}\right) \leq C_{\epsilon} h^{2 N} .
$$


Proof We first apply Tchebychev's bound :

$$
\begin{aligned}
& \mathbb{P}\left(\left|L_{\underline{0}}^{\underline{X}}\left([-h, h]^{N}\right)-L_{\underline{0}}^{\underline{X^{\prime}}}\left([-h, h]^{N}\right)\right| \geq h^{N-d H} \mid A\right) \\
& \quad \leq \frac{1}{\left(h^{N-d H}\right)^{2 k}} \mathbb{E}\left[\left(L_{\underline{0}}^{\underline{X}}\left([-h, h]^{N}\right)-L_{\underline{0}}^{\underline{X}^{\prime}}\left([-h, h]^{N}\right)\right)^{2 k} \mid A\right] .
\end{aligned}
$$

By Proposition 3 with $\frac{\epsilon}{3} 2 k>2 N$ and the bounds for $\mathbb{P}(A)$ furnished by Lemma 2 we obtain the result.

\section{Tail probabilities for $L^{*}$}

In this section we show

Proposition 4 For $C_{1.3}$ the constant of (1.6), we have

$$
\lim _{x \uparrow \infty} \frac{-\log \mathbb{P}\left(L^{*}\left([0,1]^{N}\right)>x\right)}{x^{\frac{N}{d H}}}=C_{1.3} .
$$

This is a reasonable result in the sense that for this order, there are essentially a polynomial in $x$ number of reasonable chances to obtain a large deviation for $L^{*}\left([0,1]^{N}\right)$.

The key lemmas, found in Xiao (1997) are

Lemma 3 (see Xiao 1997, Lemma 2.7) For $\gamma>0, \exists \beta, \alpha>0$ so that

$$
\mathbb{P}\left(\left|L_{\underline{u}}\left([0,1]^{N}\right)-L_{\underline{v}}\left([0,1]^{N}\right)\right| \geq|\underline{u}-\underline{v}|^{\beta}\right) \leq e^{-\alpha|\underline{u}-\underline{v}|^{-\gamma}} .
$$

We will apply the lemma with $\gamma>\frac{N}{d H}$.

Lemma 4 (see Talagrand 1995, Lemma 2.1; Xiao 1997, Lemma 3.1) There exists $C>0$ such that for $x$ large

$$
\mathbb{P}\left(\sup _{\underline{s} \in[0,1]^{N}}|\underline{X}(\underline{s})| \geq x\right) \leq e^{-C x^{2}} .
$$

Lemma 5 For each $\epsilon>0$ and $C_{1.3}$ the constant of (1.6), there exists constant $K<\infty$ so that

$$
\forall \underline{x} \in \mathbb{R}^{d}, \quad \mathbb{P}\left(L_{\underline{x}}\left([0,1]^{N}\right) \geq y\right) \leq K e^{-y \frac{N}{d H} C_{1.3}(1-\epsilon)} .
$$

Proof Let $W=L_{\underline{x}}\left([0,1]^{N}\right)$ and $Z=L_{\underline{0}}\left([0,1]^{N}\right)$. Since the centred normal law has its maximum density at $\underline{0}$, we have

$$
\mathbb{E} W^{n} \leq \mathbb{E} Z^{n}, \forall n \geq 1
$$


Using (1.6) for $n$ large, we can write for each $\epsilon>0$

$$
\begin{aligned}
& \mathbb{P}(W \geq y) \leq \frac{\mathbb{E}\left[W^{n}\right]}{y^{n}} \leq \frac{\mathbb{E}\left[Z^{n}\right]}{y^{n}} \\
& \text { which for large } n \leq \frac{(n !)^{d H / N}}{y^{n}\left(C_{1.3}-\epsilon / 100\right)^{\frac{d H n}{N}}} .
\end{aligned}
$$

The result follows by an appropriate choice of $n$ given $y$ as in Xiao (1997).

Corollary $2 \forall \gamma>\frac{N}{d H}, \quad \exists k<\infty$ so that for $x \geq 1$

$$
\mathbb{P}\left(\exists|\underline{u}|,|\underline{v}| \leq x^{\gamma}: L_{\underline{u}}\left([0,1]^{N}\right)-L_{\underline{v}}\left([0,1]^{N}\right)>1,|\underline{u}-\underline{v}| \leq \frac{1}{x^{k}}\right) \leq k e^{-x^{\frac{2 N}{d H}}}
$$

Proof It is enough to show that there exists a constant $k$ so that the inequality holds for $x$ large.

Since the local times $L_{\underline{w}}\left([0,1]^{N}\right)$ are continuous functions of the spatial variable $\underline{w}$, it is enough to show for $\bar{D}$ dense in $\left[-x^{\gamma}, x^{\gamma}\right]^{N}$ outside a set of appropriately small probability that

$$
\sup _{\substack{\underline{u}, \underline{v} \in D \\|\underline{u}-\underline{v}| \leq x}} L_{\underline{u}}\left([0,1]^{N}\right)-L_{\underline{v}}\left([0,1]^{N}\right) \leq 1 .
$$

First we choose $2^{-(l+1)} \leq x^{-k}<2^{-l}$ and consider

$$
V_{l}=2^{-l} \mathbb{Z}^{N} \cap\left[-2 x^{\gamma}, 2 x^{\gamma}\right]^{N}, \quad l=k, k+1, \ldots
$$

The event

$$
\sup _{\substack{\underline{u}, \underline{v} \in D \\ \mid \underline{\underline{v}}-\underline{\underline{v}} \leq \leq x^{-k}}} L_{\underline{u}}\left([0,1]^{N}\right)-L_{\underline{v}}\left([0,1]^{N}\right)>1
$$

is contained in

$$
\begin{gathered}
\bigcup_{\substack{\underline{u}, \underline{v} \in V_{k} \\
\underline{\underline{u}}-\underline{v} \mid \infty=2^{-k}}}\left\{\left|L_{\underline{u}}\left([0,1]^{N}\right)-L_{\underline{v}}\left([0,1]^{N}\right)\right|>\frac{1}{3}\right\} \\
\cup \bigcup_{\underline{v} \in V_{k}}\left\{\sup _{\underline{y} \in D \cap\left[\underline{v}, \underline{v}+2^{-k}\right]}\left|L_{\underline{y}}\left([0,1]^{N}\right)-L_{\underline{v}}\left([0,1]^{N}\right)\right| \geq \frac{1}{3}\right\} .
\end{gathered}
$$

The first event has probability bounded by $K 2^{k N} e^{-x^{-\frac{3 N}{d H}}}$ for some finite $K$ by Lemma 3, if $k$ is sufficiently large, which will be less than $\frac{e^{-x}-\frac{2 N}{d H}}{3}$ for $x$ large. 
It remains to consider the second event. This is contained in

$$
\bigcup_{l=k}^{\infty}\left\{\sup _{\substack{\underline{u}, \underline{v} \in V_{l} \\ \underline{\underline{u}}-\left.\underline{\underline{v}}\right|_{1}=2^{-l}}} L_{\underline{u}}\left([0,1]^{N}\right)-L_{\underline{v}}\left([0,1]^{N}\right) \geq \frac{C}{(l-k+1)^{2}}\right\}
$$

for $C=C(N)$.

And so by Lemma 3 for some $C^{\prime}<\infty$,

$$
\begin{aligned}
\mathbb{P}\left(\sup _{\substack{\left.\underline{v} \in V_{k}, \underline{v}, \underline{v}+2^{-k}\right] \\
\underline{u}}}\left|L_{\underline{u}}\left([0,1]^{N}\right)-L_{\underline{v}}\left([0,1]^{N}\right)\right|>\frac{1}{3}\right) & \leq \sum_{l=k}^{\infty} 2^{N l} x^{\gamma N} e^{-C^{\prime}\left((l-k+1)^{2} 2^{l}\right)^{3 N / d H}} \\
& \leq \frac{e^{-x^{\frac{2 N}{d H}}}}{3}
\end{aligned}
$$

if $x$ is large.

Proof of Proposition 4 Given (1.6) it is only necessarily to derive the desired upper bound for the probability. We show that the event

$$
\left\{L^{*}\left([0,1]^{N}\right)>x\right\}
$$

can be written as a union of events of appropriate probabilities.

First choose $\gamma$ so that $\gamma>\frac{N}{d H}$. Let

$$
A_{1}=\left\{\sup _{\underline{s} \in[0,1]^{N}}|\underline{X}(\underline{s})|>x^{\gamma}\right\} .
$$

By Lemma 4, we have

$$
\mathbb{P}\left(A_{1}\right) \leq e^{-C x^{2 \gamma}}
$$

Now choose $k$ according to Corollary 2 and choose a collection of points $\underline{x}_{1}, \underline{x}_{2}, \ldots, \underline{x}_{R}$ with $R \leq K_{2} x^{N(k+\gamma)}$ for some universal $K_{2}$ so that

$$
\sup _{|\underline{y}| \leq x^{\gamma}} \inf _{i}\left|\underline{y}-\underline{x}_{i}\right|<\frac{1}{x^{k}}
$$

Let

$$
A_{2}=\left\{\sup _{|\underline{y}| \leq x \gamma} \inf \left|L_{\underline{y}}\left([0,1]^{N}\right)-L_{\underline{x}_{i}}\left([0,1]^{N}\right)\right|>1\right\}
$$


and

$$
A_{3}=\left\{\exists i \leq R: L_{\underline{x}_{i}}\left([0,1]^{N}\right) \geq x-1\right\} .
$$

Then we have by Corollary 2 and Lemma 5 that for $x$ large

$$
\mathbb{P}\left(A_{2}\right) \leq k e^{-x^{\frac{2 N}{d H}}} \text { and } \mathbb{P}\left(A_{3}\right) \leq K_{2} x^{N(k+\gamma)} e^{-x^{\frac{N}{d H}} C_{1.3}(1-\epsilon)} .
$$

The result now follows from

$$
\left\{L_{\underline{x}}\left([0,1]^{N}\right)>x\right\} \subseteq A_{1} \cup A_{2} \cup A_{3}
$$

and the arbitrariness of $\epsilon$.

\section{Proof of Theorem 4}

Given the results of Sects. 2 and 3, the proof of Theorem 4 is standard. We give the proof for the interval $[-1,1]^{N}$ but it will be evident from the proof that the extension to general intervals is simply a notational exercise.

As is usual it will suffice to fix $\epsilon>0$ and to show that

$$
\limsup _{\delta \downarrow 0} \sup _{\substack{B \subseteq[-1,1] N \\|B|=\delta}} \frac{L^{*}(B)}{\psi(|B|)} \leq 1+\epsilon
$$

where $\psi(x)=x^{N-d H} \log \left(\frac{1}{x}\right)^{\frac{d H}{N}}\left(\frac{N}{C_{1.3}}\right)^{\frac{d H}{N}}$, and

$$
\liminf _{\delta \downarrow 0} \sup _{\substack{B \subseteq[-1,1] N \\|B|=\delta}} \frac{L^{*}|B|}{\psi(|B|)}>1-\epsilon
$$

We start with (7.1). Let $\sigma \ll 1$, in a manner depending on $\epsilon$ to be specified later. Consider intervals of the form

$$
I(\underline{i}, \sigma, j)=\left[\underline{i}(1+\sigma)^{-j}, \underline{i}(1+\sigma)^{-j}+(1+\sigma)^{-j}\right] \text { for } \underline{i} \in \frac{\sigma}{2} \mathbb{Z}^{N} .
$$

Let $J(j, \sigma)$ be the collection of $\underline{i}$ so that $I(\underline{i}, \sigma, j) \subset[-1,1]^{N}$. Obviously for some constant $K,|J(j, \sigma)| \leq \frac{K}{\sigma^{N}}(1+\sigma)^{j N}$.

Let $A(j, \sigma)$ be the event

$$
\bigcup_{\underline{i} \in J(j, \sigma)}\left\{L^{*}(I(\underline{i}, \sigma, j)) \geq \psi\left((1+\sigma)^{-j}\right)(1+\sigma)\right\} .
$$


Then, by stationarity of intervals,

$$
\begin{aligned}
\mathbb{P}(A(j, \sigma)) & \leq \frac{K}{\sigma^{N}}(1+\sigma)^{j N} \mathbb{P}\left(L^{*}(I(\underline{0}, \sigma, j))>\psi(1+\sigma)^{-j}(1+\sigma)\right) \\
& \leq \frac{K}{\sigma^{N}}(1+\sigma)^{-j N\left(\left(1+\frac{\sigma}{2}\right)^{\frac{N}{d H}}-1\right)} .
\end{aligned}
$$

Thus, by the first Borel-Cantelli lemma $\exists j_{0}=j_{0}(w)$ so that for $j \geq j_{0}, \quad A(j, \sigma)$ does not occur. This implies that for $\delta<(1+\sigma)^{-\left(j_{0}+2\right)}$ and $V$ an interval in $[-1,1]^{N}$ of length less than $\delta$, there exists an interval $I(\underline{i}, \sigma, j)$ containing $V$ where $\sigma \in$ $\left((1+\sigma)^{-(j+2)},(1+\sigma)^{-(j+1)}\right)$.

By the positivity of local times

$$
\begin{aligned}
L^{*}(V) \leq L^{*}(I(\underline{i}, \sigma, j)) & \leq(1+\sigma) \psi\left((1+\sigma)^{-j}\right) \\
& \leq(1+\sigma)^{1+2(N-d H)} \psi(\delta) \\
& \leq(1+\epsilon) \psi(\delta) \text { if } \sigma \text { was fixed sufficiently small. }
\end{aligned}
$$

For the inequality (7.2) we again fix $\sigma<<1$ (whose relation with $\epsilon$ will be fixed later). We consider the intervals $I(\underline{i}, \sigma, j)$ as before but now with $i \in(1+\sigma)^{\sigma j} \mathbb{Z}^{N}$. Denote by $H(\sigma, j)$ the collection of $\underline{i}$ so that $I(\underline{i}, \sigma, j) \subset[-1,1]^{N}$ and $i \in(1+\sigma)^{+\sigma j}$ $\mathbb{Z}^{N}$. It is clear that for some constant $K>0,|H(\sigma, j)| \geq K(1+\sigma)^{\bar{N}(1-\sigma)}$.

The point is that if $K(\underline{i}, \sigma, j)$ is the cube with side length $\frac{(1+\sigma)^{-j(1-\sigma)}}{3}$ and the same centre as $I(\underline{i}, \sigma, j)$ then the $K(\underline{i}, \sigma, j)$ are disjoint for $\underline{i} \in H(\sigma, \underline{j})$.

We suppose, enlarging the probability space if necessary, that we have white noises $Z^{i}, \underline{i} \in H(\sigma, j)$, independent of each other and of the underlying white noise $W$. We define the comparison processes

$$
\begin{aligned}
X^{\underline{i}}(\underline{t})= & C_{N, H} \int_{K(\underline{i}, \sigma, j)}\left(\frac{1}{|\underline{x}-\underline{t}|^{\frac{N}{2}-H}}-\frac{1}{|\underline{x}|^{\frac{N}{2}-H}}\right) d \dot{W}(\underline{x}) \\
& +C_{N, H} \int_{K(\underline{i}, \sigma, j)^{c}}\left(\frac{1}{|\underline{x}-\underline{t}|^{\frac{N}{2}-H}}-\frac{1}{|\underline{x}|^{\frac{N}{2}-H}}\right) d \dot{Z}^{\underline{i}}(\underline{x}) .
\end{aligned}
$$

By the disjointness of the sets $K(\underline{i}, \sigma, j)$ and the independence of the white noises $Z^{i}-$, the processes $X^{i}$ are independent. We define the events

$$
\left.\left.A(\underline{i}, j)=\left\{L_{X^{\underline{i}}(\underline{\underline{i}} \underline{\underline{i}})}^{X^{\underline{i}}}(I(\underline{i}, \sigma, j)) \geq \psi(1+\sigma)^{-j}\right)(1-\epsilon)+(1+\sigma)^{-j}\right)^{N-H d}\right\},
$$

where $\underline{t} \underline{i}$ is the central point of interval $I(\underline{i} . \sigma, j)$. By the definition of constant $C_{1.3}$ and thus the function $\psi$, we have for all $j$ large,

$$
\forall \underline{i}, \quad \mathbb{P}(A(\underline{i}, j)) \geq(1+\sigma)^{-j N(1-\epsilon / 2)}
$$


Then, provided $\sigma<\epsilon / 4<<1$, for $j$ large

$$
\mathbb{P}\left(\bigcap_{I \in H(\sigma, j)} A(\underline{i}, j)^{c}\right) \leq\left(1-(1+\sigma)^{j N(1-\epsilon / 2)}\right)^{K(1+\sigma)^{N j(1-\sigma)}} \leq e^{-(1+\sigma)^{j \epsilon / 4}}
$$

But now consider the events

$$
B(\underline{i}, j)=\left\{\left|L_{X \underline{\underline{i}}(\underline{\underline{t}}-)}^{\left.X^{\underline{i}}\right)}(I(\underline{i}, \sigma, j))-L_{X(\underline{t} \underline{\underline{i}})}^{X}(I(\underline{i}, \sigma, j))\right| \geq(1+\sigma)^{j(N-H d)}\right\} .
$$

By Proposition 3, $\mathbb{P}\left(\cup_{\underline{i}} B(\underline{i}, j)\right)<(1+\sigma)^{j N}(1+\sigma)^{-2 j N} \leq(1+\sigma)^{-j N}$ for $j$ large. So we have

$$
\begin{aligned}
& \mathbb{P}\left(\sup _{\underline{i}} L^{*}(I(\underline{i}, \sigma, j)) \geq \psi\left((1+\sigma)^{-j}\right)(1-\epsilon)\right) \\
& \geq \mathbb{P}\left(\sup _{\underline{i}} L_{X\left(\underline{t}^{i}\right)}^{X}(I(\underline{i}, \sigma, j)) \geq \psi\left((1+\sigma)^{-j}\right)(1-\epsilon)\right) \\
& \geq 1-\mathbb{P}\left(\bigcap_{\underline{i}} A(\underline{i}, j)^{c}\right)-\mathbb{P}\left(\bigcup_{\underline{i}} B(\underline{i}, j)\right) .
\end{aligned}
$$

This last bound is easily seen to be greater than $1-2(1+\sigma)^{-j}$ for $j$ large.

Thus by the first Borel-Cantelli lemma we have a.s. for all $j$ large

$$
\sup _{\substack{B \subset[-1,1]^{N} \\|B|=(1+\sigma)^{-j}}} L^{*}(B) \geq \psi(|B|)(1-\epsilon) .
$$

For general $\delta$ small enough we can find $j$ so that $\delta \in\left[(1+\sigma)^{-(j+1)},(1+\sigma)^{-j}\right)$ and so by monotonicity of local times we have (for $\delta$ small)

$$
\sup _{\substack{B \subset[-1,1]^{N} \\|B|=\delta}} L^{*}(B) \geq \psi(|B| /(1+\sigma))(1-\epsilon) .
$$

The result follows immediately from the arbitrariness of $\sigma$ and $\epsilon$.

\section{References}

Adler RJ (1981) The geometry of random fields. Wiley, New York

Baraka D, Mountford T (1997) A law of iterated logarithm for fractional Brownian motions (to appear)

Baraka D, Mountford T (2008) The exact Hausdorff measure of the zero set of fractional Brownian motion (to appear)

Embrechts P, Maejima M (2002) Selfsimilar processes. Princeton University Press, Princeton

Geman D, Horowitz J (1980) Occupation densities. Ann Probab 8:1-67 
Geman D, Horowitz J, Rosen J (1984) A local time analysis of intersections of Brownian paths in the plane. Ann Probab 12:86-107

Kasahara Y (1978) Tauberian theorems of exponential type. J Math Kyoto Univ 18:209-219

Kasahara Y, Kôno N, Ogawa T (1999) On tail probability of local times of Gaussian processes. Stoch Process Appl 82:15-21

Mountford T (2007) Level sets of multiparameter stable processes. J Theoret Probab 20:25-46

Mountford T, Nualart E (2004) Level sets of multiparameter Brownian motions. Electron J Probab 9: 594-614

Mountford T, Shieh NR, Xiao Y (2008) The tail probabilities for local times of fractional Brownian motion (to appear)

Pitt LD, Tran LT (1979) Local sample path properties of Gaussian fields. Ann Probab 7:477-493

Ross S (1996) Initiation aux Probabilités. Presses Polytechniques et Universitaires Romandes, Lausanne

Rogers CA (1998) Hausdorff measures. Cambridge University Press, Cambridge

Samorodnitsky G, Taqqu MS (1994) Stable non-Gaussian random processes: stochastic models with infinite variance, stochastic modeling. Chapman \& Hall, New York

Talagrand M (1995) Hausdorff measure of trajectories of multiparameter fractional Brownian motion. Ann Probab 23:767-775

Talagrand M (1998) Multiple points of trajectories of multiparameter fractional Brownian motion. Probab Th Rel Fields 112:545-563

Xiao Y (1997) Hölder conditions for the local times and the Hausdorff measure of the level sets of Gaussian random fields. Probab Th Rel Fields 109:129-157

Xiao Y (2006) Properties of local-nondeterminism of Gaussian and stable random fields and their applications. Ann Fac Sci Toulouse Math (6) 15:157-193

Xiao Y (2007) Strong local nondeterminism of Gaussian random fields and its applications. In: Lai T-L, Shao Q-M, Qian L (eds) Asymptotic theory in probability and statistics with applications. Higher Education Press, Beijing, pp 136-176 